\title{
INVESTIGACIONES ARQUEOLÓGICAS EN TORNO A LOS PRIMEROS REGISTROS DE ARTE RUPESTRE EN MORRO VILCÚN
}

\section{ARCHAEOLOGICAL INVESTIGATIONS OF THE FIRST RECORDED ROCK ART SITE ON MORRO VILCÚN}

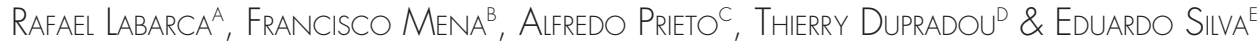

Se presentan los resultados de un proyecto FONDART orientado a describir sistemáticamente pinturas y grabados rupestres de tres cuevas emplazadas en el litoral de la Patagonia septentrional chilena, recientemente descubiertas. Se registraron 47 pinturas rojas y nueve grabados lineales, con variaciones en la distribución de los motivos, tanto al interior de cada cueva como entre ellas. Aunque no se alcanzó la base de los depósitos, los trabajos realizados proveen el marco inicial para futuros estudios.

Palabras clave: arte rupestre, ocupaciones costeras, Patagonia, sondeos arqueológicos, arqueología comunitaria.

This paper presents the results of a FONDART project aimed at describing the first rock art found on the coasts of the Northern Patagonian channels. The project involved exhaustive, systematic records of the wall paintings and engravings found. Forty-seven paintings were recorded (mostly lines, dots and spots, all in red) as well as nine engravings. Some distributional patterns are noted. Although the bottom of the depositional sequence could not be reached and the answer to many research questions (e .g. the cultural affiliation of the occupants) is still pending, analyses of both surface and subsurface records (rock art and mobile items) provided enough information to move beyond vague speculation and formulate basic hypotheses that can be tested in future research.

Keywords: Rock art, coastal ocupations, Patagonia, archaeological test pits, community archaeology.

\section{INTRODUCCIÓN}

Tradicionalmente, ha habido una gran distancia entre los rumores locales y la investigación científica conducida por afuerinos, y de no ser porque la prehistoria de Chiloé continental permanece casi desconocida, es probable que ni siquiera prestáramos oídos a los datos sobre pinturas rupestres en las cercanías de Chaitén (Equipo PAT 2012). La decisión de comprobar en terreno la veracidad de tales rumores, sin embargo, condujo al "descubrimiento" (en el sentido de documentación académica, al menos) de un notable conjunto de cuevas en el morro Vilcún y a su posterior difusión escrita (Mena et al. 2011). Al año siguiente -gracias a un proyecto FONDART- se pudo realizar investigaciones más sistemáticas en cuatro de esas cuevas, las que se tradujeron inicialmente en charlas a la comunidad, además de una exposición fotográfica con fines artísticos y educativos en Chaitén y Coyhaique. Con esta publicación académica -en que damos a conocer los resultados del análisis de las colecciones recuperadas en un marco más técnico y especializado-, terminamos este proyecto, esperando motive otras investigaciones en la zona.

A Rafael Labarca, Laboratorio de Paleoecología, Instituto de Ciencias Ambientales y Evolutivas, Universidad Austral de Chile, Casilla 567, Valdivia, Chile, email: r.labarca.e@gmail.com

B Francisco Mena, Centro de Investigación en Ecosistemas de la Patagonia (cieP), conicyt Regional R101003, Coyhaique, Chile, email: francisco.mena@ciep.cl

C Alfredo Prieto, Centro de Estudios del Hombre Austral, Instituto de la Patagonia, Universidad de Magallanes, Punta Arenas, Chile, email: alfredo.prieto@umag.cl

D Thierry Dupradou, Quillota 150, Punta Arenas, Chile, email: thdupradou@voila.fr

E Eduardo Silva, Rossini 7059, Las Condes, Santiago, Chile, email: eduardo.silvac1@gmail.com 
Aun cuando el interés del proyecto fue, en principio, gatillado por la presencia de arte rupestre -acerca de cuya existencia en este territorio no había sospechas-, la escasa y segmentada información disponible sobre la prehistoria del área hace que cualquier investigación signifique un aporte sustantivo. De ahí que un fenómeno llamativo como el arte rupestre y su valor patrimonial y turístico se pueda constituir en una especie de "puerta de entrada" a la investigación prehistórica de la región, despertando preguntas sobre la cronología de las ocupaciones, la subsistencia, el asentamiento y otros problemas que no solo rebasan el arte rupestre, sino nuestras propias investigaciones. Considerando lo anterior, realizamos algunos sondeos acotados y obtuvimos un par de fechados radiocarbónicos y algunos materiales culturales -fragmentos cerámicos y desechos líticos- y ecofactuales. De esta manera, el presente trabajo tiene por objetivo principal entregar los resultados de nuestros trabajos arqueológicos efectuados tanto en terreno como en laboratorio, los cuales son discutidos y contextualizados a la luz de la evidencia regional.

\section{LOCALIZACIÓN Y ASPECTO FÍSICO DE LAS CUEVAS}

En una etapa inicial del estudio se dio a conocer la existencia de tres cuevas (Mena et al. 2011), pero en el presente artículo incluimos una cuarta, identificada en esta nueva etapa de trabajo de campo. Los cuatro reparos rocosos (cuevas Chica, Mediana, Grande y Alta) son cercanos entre sí y se emplazan en la cara sur del morro Vilcún, un antiguo cuello volcánico de 420 $\mathrm{m}$ de altitud ubicado $3,3 \mathrm{~km}$ al No de la localidad de Santa Bárbara, próxima a la ciudad de Chaitén (región de Los Lagos; fig. 1). Las cavidades corresponderían a burbujas en este cuello riolítico, erosionadas por el avance del mar y la infiltración constante de agua. Respecto de la vegetación, la localidad se encuentra inserta en la región vegetal del bosque Siempreverde y de las Turberas (Gajardo 1994).

Las cuevas Chica [18G E0677316; N5255015] y Mediana [18G E0677238; N5254987] (figs. 2a y 2b) están separadas solo por una gruesa columna que se extiende hasta aproximadamente cuatro metros antes de la línea de goteo. Ambas poseen una orientación general Ns, ubicándose a cuatro metros de distancia de la línea de alta marea y a apenas $3,2 \mathrm{~m}$ sobre esta.

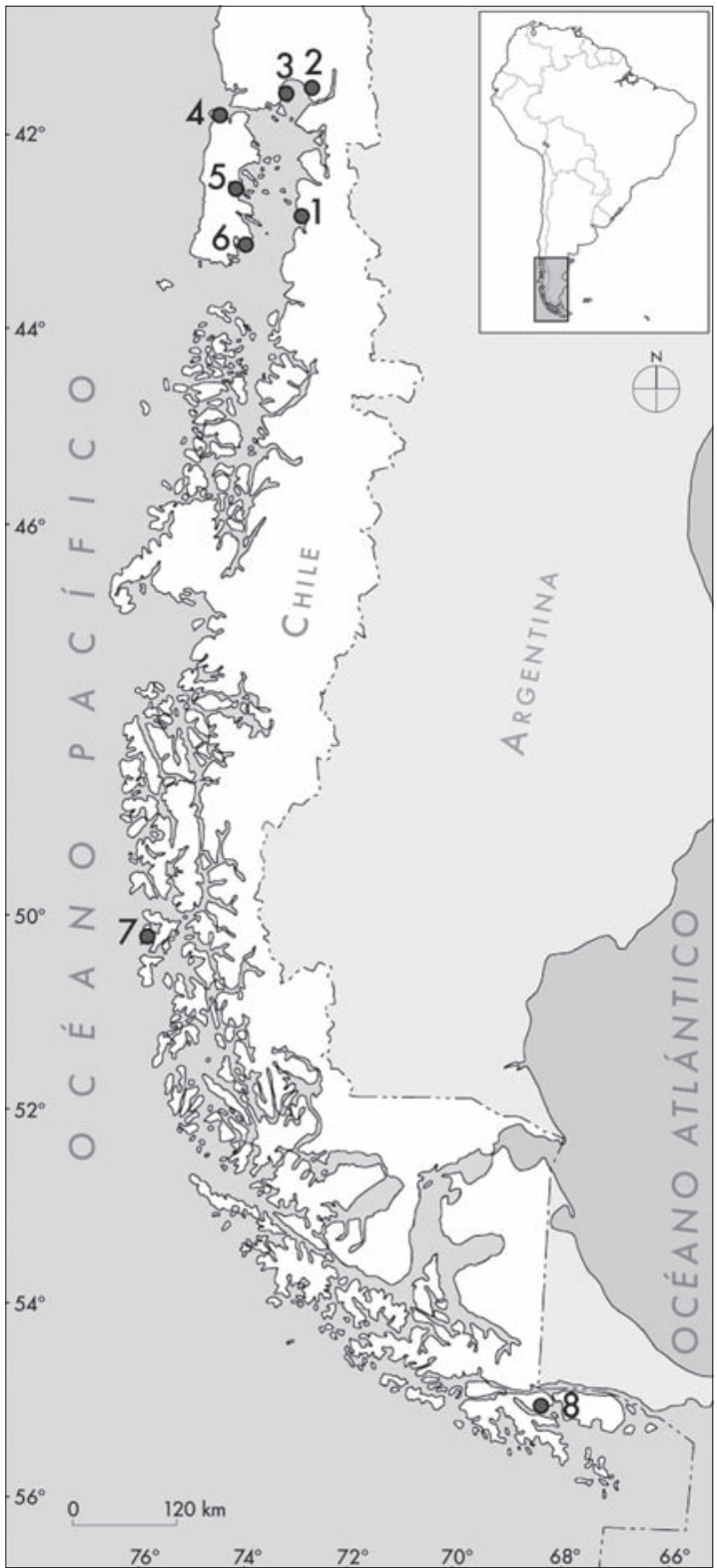

Figura 1. Ubicación de los sitios arqueológicos mencionados en el texto: 1) Morro Vilcún; 2) Piedra azul; 3) Huenquillahue 2; 4) Puente Quilo 1;5) Conchal Gamboa; 6) Yaldad 2; 7) Cueva Pacifico; 8) Cueva Martín González. Figure 1. Location of the archeological sites mentioned in the text: 1) morro Vilcún; 2) Piedra Azul; 3) Huenquillahue 2; 4) Puente Quilo 1; 5) conchal Gamboa; 6) Yaldad 2; 7) cueva Pacífico; 8) cueva Martín González. 


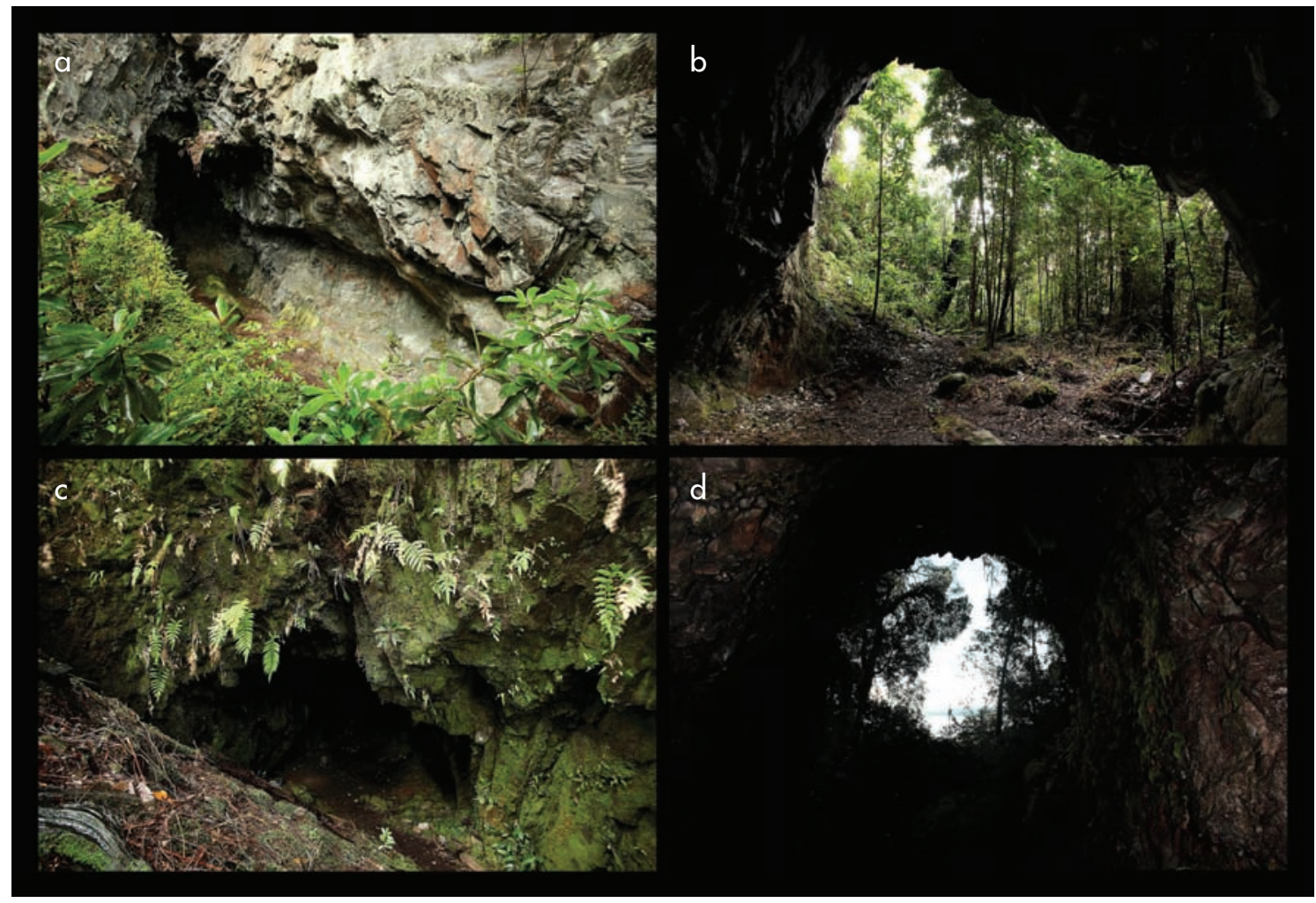

Figura 2. Las cuevas del morro Vilcún: a) cueva Chica; b) cueva Mediana; c) cueva Grande; d) cueva Alta. Figure 2. The caves of morro Vilcún: a) Small cave; $\boldsymbol{b}$ ) Medium cave; $c$ ) Large cave; $d$ ) High cave.

La cueva Chica (fig. 2a) es de 5,2 $\mathrm{m}$ de largo, 3,7 $\mathrm{m}$ de ancho y una altura de $3 \mathrm{~m}$ sobre el piso en la línea de goteo. A unos dos metros de esta línea, un pequeño espigón que emerge desde el piso asciende hasta formar dos pequeñas recámaras rocosas en la parte posterior, de no más de $1,5 \times 2$ m cada una. Por su parte, la cueva Mediana (fig. 2 b y 3 ) cuenta con una abertura de 8,5 $\mathrm{m}$ de ancho máximo y $6,8 \mathrm{~m}$ de alto sobre el piso en la línea de goteo, en tanto su largo máximo es de 11,6 m. Presenta una cámara principal (oeste) de $39,7 \mathrm{~m}^{2}$ más un angosto apéndice ubicado al este (cámara este). En la superficie de la parte anterior de la cueva se hallaron numerosos troncos y maderas asociados a dos fogones delimitados por rocas al norte, además de basura subactual y restos de moluscos, lo que indica su utilización hasta la actualidad.

La cueva Grande [18G 0677304; 5255011] (figs. 2c y 4), emplazada a unos $10 \mathrm{~m}$ al oeste de la cueva Mediana, corresponde a una gran oquedad de 17,6 m de largo a la línea de goteo, 3,8 $\mathrm{m}$ de alto máximo al piso actual y apenas 4,7 $\mathrm{m}$ de ancho máximo. Su orientación es Ns y se ubica a $45 \mathrm{~m}$ de la línea de alta marea y a 6,4 $\mathrm{msnm}$. El acceso se encuentra parcialmente sepultado por un antiguo derrumbe en su frente oeste, el que se interna hacia la cueva y que dejó tan solo un pequeño pasillo de acceso. En su parte anterior, la cueva cuenta con una cámara principal, que constituye cerca del 70\% de la superficie bajo reparo del sitio y que conduce a un pequeño pasillo cuyo piso posee mayor altura que el segmento recién comentado. En promedio, esta porción es de $1,5 \mathrm{~m}$ de ancho y $1,3 \mathrm{~m}$ de alto, dimensiones que disminuyen paulatinamente hacia el interior. Hacia este y oeste exhibe a lo menos cuatro pequeñas recámaras de muy baja altura (aprox. 0,5 $\mathrm{m}$ ) y de profundidad no determinada. En superficie, el sector trasero ostenta una matriz carbonosa que incluye gran cantidad de valvas de moluscos bien conservadas, sobre todo lapas (Fisurella spp.), tégulas (Tegula atra) y locos (Concholepas concholepas). Al menos cinco excavaciones ilegales de distinto tamaño se observaron dentro de la cueva. 


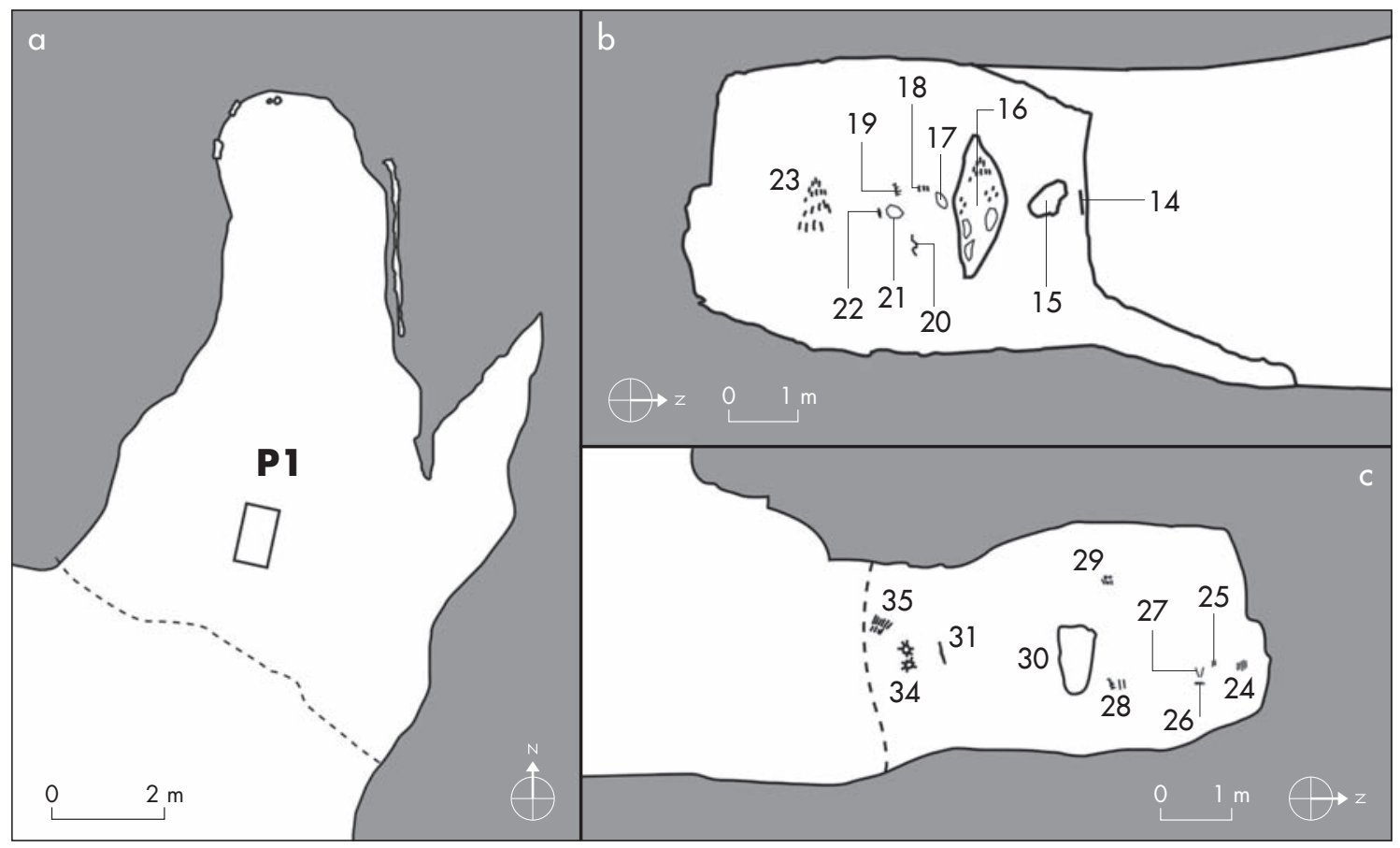

Figura 3. Planta y perfil de la cueva Mediana con la ubicación de los pozos de sondeo y pinturas más representativas: a) planta general; b) cámara oeste, perfil este; c) cámara oeste, perfil oeste. Figure 3. Floor plan and profile of the Medium cave showing location of test pits and most representative paintings: a) general floor plan; $b$ ) west chamber, eastern profile; $c$ ) west chamber, western profile.

Finalmente, la cueva Alta [aprox. 18G E0677320; N5255053] (fig. 2d) se emplaza en un pequeño promontorio al noreste de las cuevas Chica y Mediana, a unos $16 \mathrm{msnm}$. A diferencia de las anteriores, posee una orientación NE-so. Tiene un largo de 19,5 m, un ancho de 2,7 $\mathrm{m}$ y una altura cercana a los $2 \mathrm{~m}$ en la línea de goteo. Un derrumbe en su sector medio permite dividirla en dos sectores, de los cuales solo el anterior recibe luz solar. Presenta restos fragmentados de moluscos en superficie (al menos, tégulas y locos) sin evidencias actuales de alteración antrópica.

\section{MÉTODOS}

Las actividades en terreno se dividieron en: documentación fotográfica, registro del arte rupestre y levantamiento de plantas y perfiles, además de sondeos arqueológicos. En cuanto a la primera, se tomaron fotografías de cada motivo rupestre. Luego, algunas de ellas fueron tratadas con el programa D-Stretch@ (Harman 2016), con el objeto de resaltar motivos y así evitar interpretaciones y distorsiones vinculadas con la evaluación del arte rupestre únicamente a través de dibujos. Las imágenes que se muestran en este trabajo, sin embargo, no fueron manejadas con el programa mencionado. Este registro fue complementado por dibujos generales, con el fin de constatar la disposición de los motivos y su asociación contextual. Se consideró como "unidad" un motivo simple o compuesto (panel o conjunto), definido de modo relativamente arbitrario y subjetivo como aquello perceptible en una mirada y susceptible de haberse hecho por una persona en un momento (Leroi-Gourhan 1968; Gradín 1978).

A partir de un eje sagital, se efectuaron levantamientos (brújula y huincha) de planta y perfil de las paredes de las cuatro cuevas estudiadas. En cada uno de estos se incluyó la ubicación de los distintos motivos, utilizando la misma nomenclatura de los registros de campo y fotografías. En consecuencia, el objetivo de esta labor ha sido registrar y presentar las manifestaciones halladas, y no tiene ninguna pretensión interpretativa ni responde a una perspectiva teórica en particular.

Para contextualizar las manifestaciones rupestres, se llevaron a cabo tres intervenciones estratigráficas 


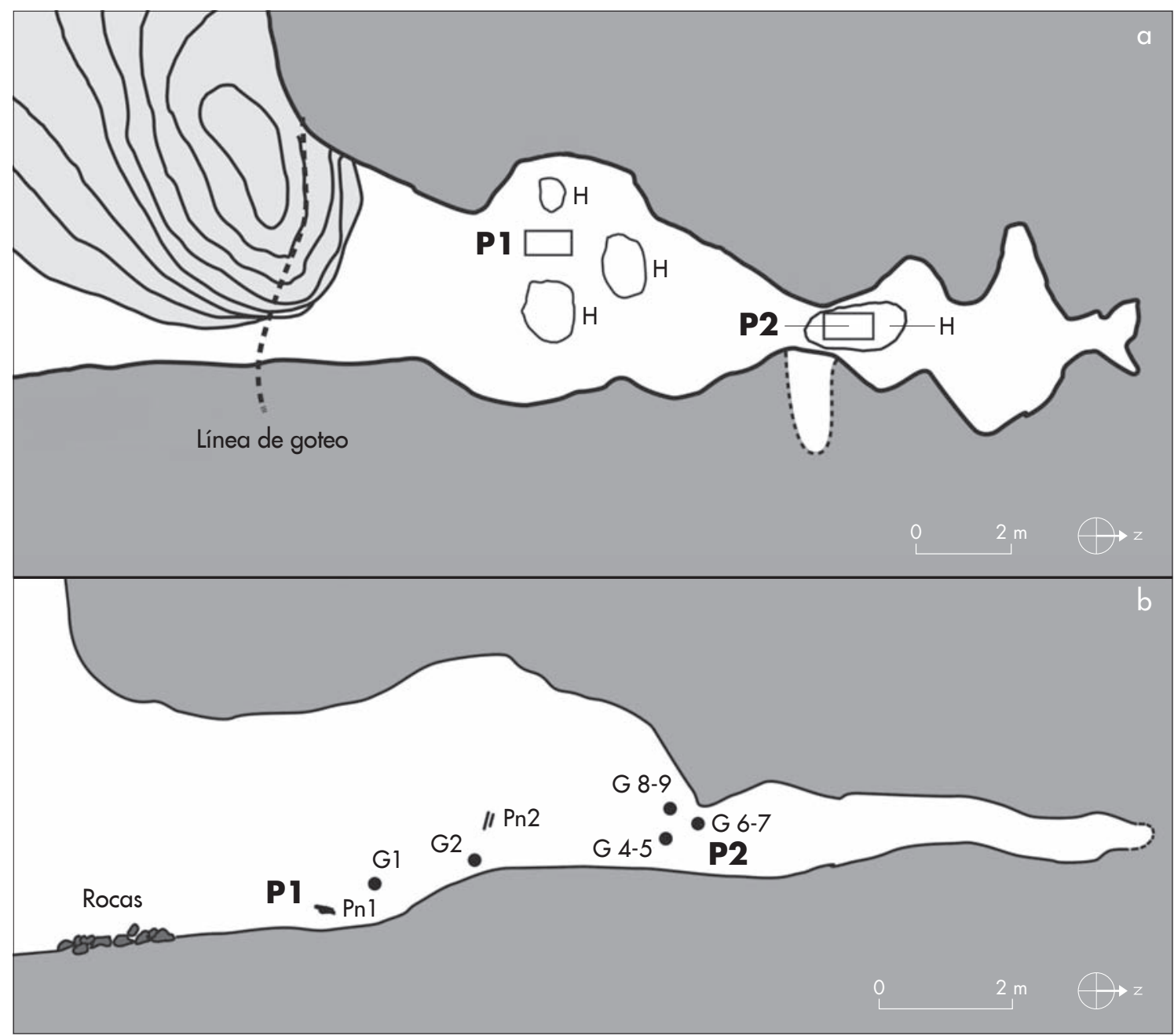

Figura 4. Planta y perfil de la cueva Grande con la ubicación de los pozos de sondeo y representaciones rupestres: H) "huaqueo" (pozo de saqueo); Pn) pinturas; G) grabado. Figure 4. Floor plan and profile of the Large cave showing locations of test pits and rock art representations: H) "huaqueo" (test pit yielding artifacts); Pn) paintings; G) engraving.

acotadas en las cuevas Mediana y Grande. La cueva Chica no fue sondeada en razón de su escaso potencial estratigráfico y la ausencia de motivos rupestres, mientras que la cueva Alta corrió la misma suerte debido a que excedía el tiempo propuesto. Los pozos de sondeo tuvieron dimensiones de $1 \times$ x $0,5 \mathrm{~m}$ y fueron excavados en niveles artificiales de $10 \mathrm{~cm}$, considerando además la estratigrafía de los depósitos. Los sedimentos fueron harneados en agua con malla de $0,3 \mathrm{~cm}$. Se realizaron dibujos de planta y de perfiles, tomando muestras de moluscos y crustáceos selectivamente en cada nivel con el objeto de obtener una aproximación a su diversidad. De igual manera, en cada nivel se extrajeron muestras de carbón para fechados absolutos. Todos los materiales culturales fueron embolsados y etiquetados de acuerdo a su procedencia estratigráfica.

Las actividades de análisis, por su parte, incluyeron la identificación taxonómica y asignación anatómica de los restos óseos (uso de guías y esqueletos de referencia; Falabella et al. 1995), cuantificaciones generales (Grayson 1984) y registro de modificaciones naturales y antrópicas (Lyman 1994). En lo que respecta al análisis artefactual (cerámico y lítico), se tomaron algunas medidas básicas, se realizó una descripción general y se observaron atributos funcionales, tecnológicos y estilísticos (Andrefsky 2006; Rice 2006). 
Tabla 1. Resumen de pinturas rupestres registradas en el morro Vilcún: CM) cueva Mediana; CG) cueva Grande; CA) cueva Alta; CaE) cámara este; $\mathrm{CaW}$ ) cámara oeste; $\mathrm{PE}$ ) pared este; PW) pared oeste. Table 1. Summary of rock paintings recorded at Morro Vilcún. CM) Medium Cave; CG) Large Cave; CA) High Cave; CaE: East Chamber; CaW: West Chamber; PE) East Wall; PW) West Wall, Large Cave; P2 CG) Pit 2 in Large Cave; P1 CM) Pit 1 in Medium Cave.

\begin{tabular}{|c|c|c|c|c|c|c|c|c|c|}
\hline \multicolumn{7}{|c|}{$\mathrm{CM}$} & \multirow{2}{*}{$\begin{array}{c}\text { CG } \\
\text { PW }\end{array}$} & \multirow{2}{*}{$\frac{\mathrm{CA}}{\mathrm{PE}}$} & \multirow[t]{2}{*}{ Total } \\
\hline & $\mathrm{CaE} / \mathrm{PE}$ & $\mathrm{CaE} / \mathrm{PW}$ & $\mathrm{CaW} / \mathrm{PE}$ & $\mathrm{CaW} / \mathrm{PW}$ & Total CM & PW & & & \\
\hline Líneas & 2 & & 7 & 4 & 13 & 1 & 2 & & 16 \\
\hline Puntos & & & & 1 & 1 & & & & 1 \\
\hline Manchones & 5 & & 3 & 2 & 10 & 1 & & 1 & 12 \\
\hline Manchones/flecha & 1 & & & & 1 & & & & 1 \\
\hline Manchones/línea & 5 & & & & 5 & & & & 5 \\
\hline Manchones/punto & & & 1 & & 1 & & & & 1 \\
\hline Línea/punto & & & & & & & 2 & & 2 \\
\hline Punto/línea/"peine" & & & & 1 & 1 & & & & 1 \\
\hline Rombo & 1 & & & & 1 & & & & 1 \\
\hline Cuadrado & 1 & & & 1 & 2 & & & & 2 \\
\hline Círculo & & & & 1 & 1 & & & & 1 \\
\hline Mano? & 1 & & & & 1 & & & & 1 \\
\hline "Peine" & & & 1 & & 1 & & & & 1 \\
\hline Zigzag & & & 1 & & 1 & & & & 1 \\
\hline Chevron & & & & 1 & 1 & & & & 1 \\
\hline Total & 16 & & 13 & 11 & 40 & 2 & 4 & 1 & 47 \\
\hline
\end{tabular}

\section{RESULTADOS}

\section{Arte rupestre}

Un total de 47 pinturas rupestres y nueve grabados fueron documentados en las cuevas Mediana, Grande y Alta. La cueva Chica, por su parte, no exhibe manifestaciones de ningún tipo. Un dato importante es que todos ellos -pinturas y grabados- se encuentran exclusivamente en las paredes de los reparos.

La mayoría de los motivos pintados se concentran en la cueva Mediana ( $\mathrm{N}=40 ; 85,1 \%$; fig. 3 ), en desmedro de las cuevas Grande ( $\mathrm{N}=2 ; 4,2 \%$; fig. 4$)$ y Alta ( $\mathrm{N}=5 ; 10,6 \%)$. Por su parte, la totalidad de los grabados se ubican en la cueva Grande (N=9; fig. 4). Se trata sobre todo de líneas de distinta extensión y grosor, y "manchones", es decir, áreas acotadas cubiertas con pigmento de morfología irregular (figs. 5c y 6a). En menor medida, se registraron motivos más definidos, como rombos, círculos, cuadra- dos, chevrones, "peines" (conformados por una línea principal con trazos transversales paralelos) (figs. 5 a y d; figs. 6 b y c) y un posible positivo de mano (figura 5 b; tabla 1). Todas fueron realizadas en rojo, presuntamente un óxido de fierro que no se encuentra en forma natural en el tipo de roca que constituye el morro. Las líneas se disponen aisladas o formando grupos, mientras que su extensión y espesor varían. De esta manera, se advierten trazos de $3 \mathrm{~cm}$ hasta $15 \mathrm{~cm}$ de longitud, mientras que los espesores oscilan entre $4 \mathrm{~mm}$ y $3 \mathrm{~cm}$, lo que sugiere distintas técnicas de impresión. Los manchones, en tanto, pueden corresponder a diseños alterados con el paso del tiempo, pero también a motivos en sí mismos, ya que algunas de estas manifestaciones ostentan coloraciones muy intensas y sin signos de intemperización.

El emplazamiento de las pinturas es variable y no se aprecian tendencias claras en relación con su ubicación en las paredes. La altura respecto del piso actual es también bastante diversa, al punto que se observan motivos desde 


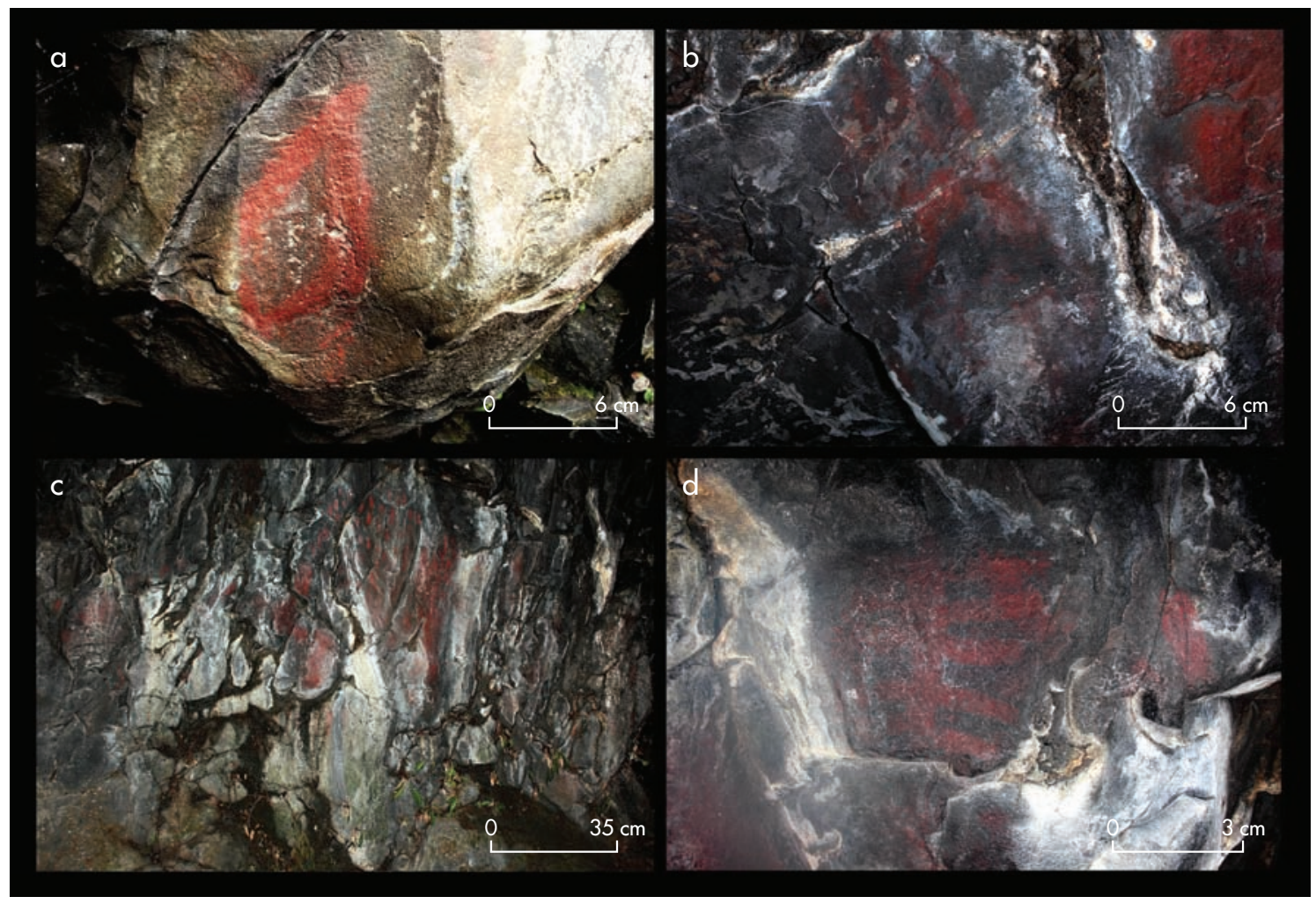

Figura 5. Pinturas rupestres de la cueva mediana: a) motivo $\mathrm{n}^{\circ} 1$ (rombo); b) motivo $\mathrm{n}^{\circ} 12 \mathrm{~b}$ (¿positivo de mano?); $\mathrm{c}$ ) motivo $\mathrm{n}^{\circ} 16$ (líneas y manchones); d) motivo $n^{\circ} 19$ ("peine"). Figure 5. Rock paintings in the Medium cave: a) motif $n^{\circ} 1$ (diamond); $\boldsymbol{b}$ ) motif $n^{\circ} 12 b$ (¿hand print?); c) motif $n^{\circ} 16$ (lines and spots); d) motif $n^{\circ} 19$ ("comb").

casi el nivel del suelo hasta más de $2 \mathrm{~m}$ de altura (fig. 3). $\mathrm{Al}$ respecto, la presencia de pinturas muy cercanas al piso actual en las cuevas con depositación estratigráfica, como la cueva Grande (fig. 4) y posiblemente la cueva Alta, hace suponer que otras manifestaciones rupestres también se encuentran sepultadas. Son recurrentes las agrupaciones de motivos no superpuestos entre sí, de entre los cuales la asociación línea/manchón destaca como la más popular. En este sentido, las relaciones entre los motivos parecen ser circunstanciales, pues no se aprecia una intención de generar representaciones mayores a partir de conjuntos de motivos, como sí ocurre en contextos de cazadores terrestres patagónicos (Podestá et al. 2005). Tentativamente, creemos que cada motivo es independiente y fruto de una sola acción creativa. De manera excepcional, solo en la cueva Grande se observaron grabados confeccionados mediante piqueteado y raspado de profundidad variable $(\mathrm{N}=9)$, en forma de círculos o subcírculos y con trazos en su interior (figs. 4 y 6d). Por último, es importante señalar que la mayoría de las pinturas se encuentran vandalizadas y sujetas a cierta intervención por el graffitti moderno.

\section{Sondeos estratigráficos}

\section{Cueva Mediana}

El pozo 1 (fig. 7a) se emplazó en el sector central de la cueva Mediana, a 1,6 $\mathrm{m}$ de la línea de goteo, y alcanzó una profundidad máxima de $40 \mathrm{~cm}$ hasta la roca base. Se registraron un total de cuatro estratos o capas. La capa 1 corresponde a una matriz orgánica (humus) muy húmeda, con algunos clastos angulares posiblemente caídos desde el techo. Presenta entre 3 y $6 \mathrm{~cm}$ de potencia. Bajo esta capa, y entre los 6 y $11 \mathrm{~cm}$ de profundidad, se documentó la capa 2a, compuesta por una matriz carbonosa, intercalada con lentes de ceniza color crema y gris. Presenta restos de moluscos muy 


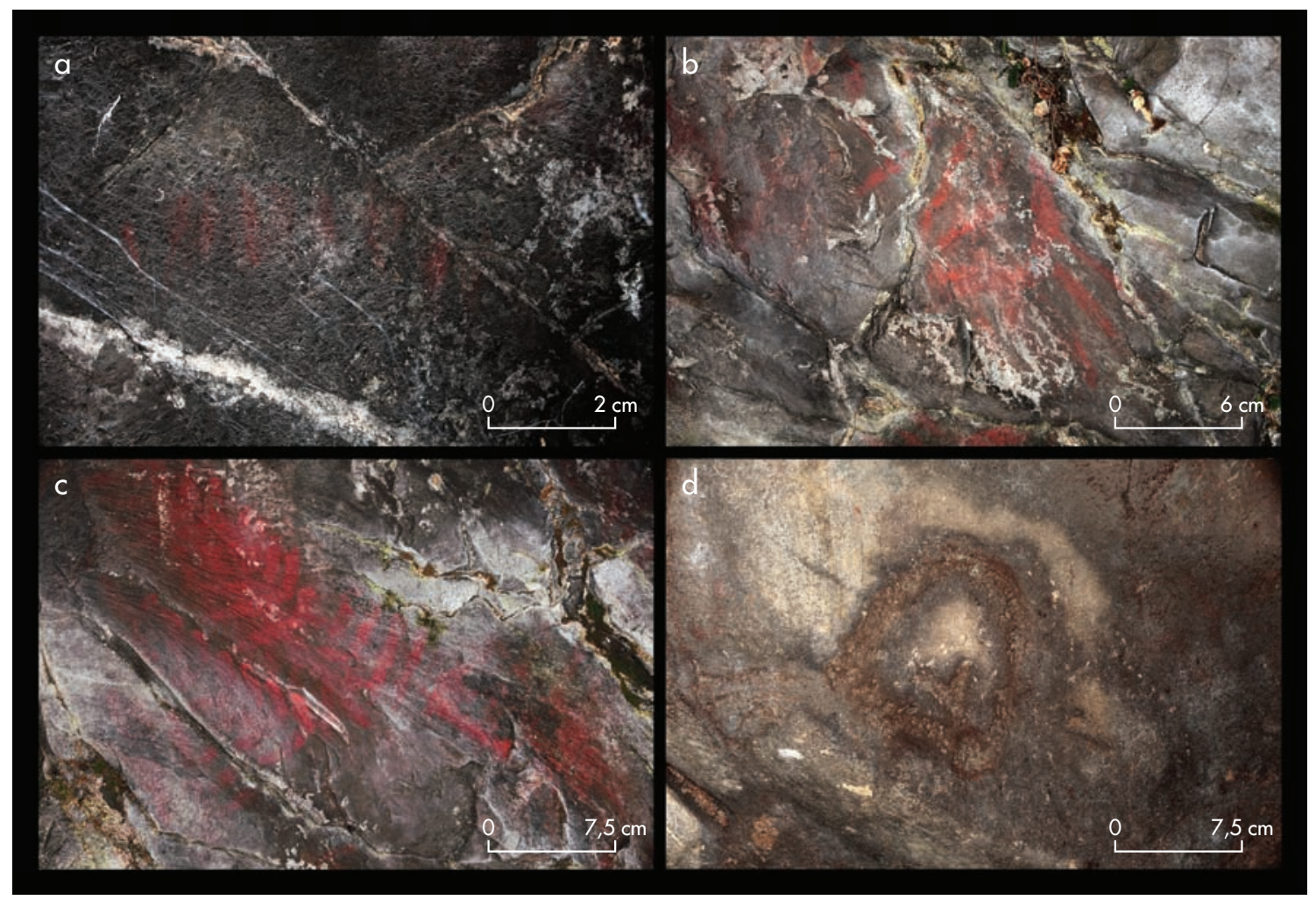

Figura 6. Pinturas y grabados de la cueva Mediana y Grande: a) motivo $\mathrm{n}^{\circ} 26$ (líneas); b) motivo $\mathrm{n}^{\circ} 32$ (círculo con trazos divergentes); c) motivo $n^{\circ} 35$ (chevrones); d) grabado 2. Figure 6. Paintings and engravings in the medium and Large caves: $\boldsymbol{a}$ ) motif $n^{\circ} 26$ (lines); $\boldsymbol{b}$ ) motif $n^{\circ} 32$ (circle with diverging lines); c) motif $n^{\circ} 35$ (chevrons); d) engraving 2.

fragmentados y exoesqueletos de erizo (Echinoidea indet.), así como también material histórico, sobre todo clavos y porciones de metal. Luego se halla la capa $2 b$, entre los 11-13 y 20-26 cm de profundidad, la cual se diferencia del estrato anterior por no contener lentes de ceniza, pues corresponde a una matriz carbonosa bastante homogénea. Asimismo, en ella se observan valvas comparativamente más completas de loco, lapa, mitílidos, restos de poliplacóforos (Poliplacophora indet.) y exoesqueletos de erizo. En esta capa se recuperaron dos fragmentos cerámicos. En el sector central de la unidad, y cerca de $l o s 16 \mathrm{~cm}$ de profundidad, se documentó un fogón de morfología ovalada (30 x 40 $\mathrm{cm}$ ), denominado Rasgo 1, compuesto por una matriz cenicienta y tierra quemada en su base. El final de este coincide con el fin de la capa $2 \mathrm{~b}$.

Más abajo, entre los 17 y $26 \mathrm{~cm}$ de profundidad, se halla la capa 3 , que corresponde a una matriz de arena gruesa, color negruzco y con alto contenido de carbón. La base de este estrato coincidió, en el sector central, con el inicio de la roca base de la cueva. Inmediatamente bajo la capa 3, y descansando por completo sobre la base de la cueva, se encuentra la capa o estrato 4 , que alcanza hasta los $34 \mathrm{~cm}$ de profundidad. Se trata de una matriz muy húmeda, carbonosa, con pequeños lentes cenicientos, menor contenido de arena, presencia de clastos, guijarros y conchas fragmentadas similares a las documentadas en los estratos precedentes. Una muestra de carbón proveniente de esta capa arrojó una edad de $190 \pm 20$ AP (UgAMS 11723).

\section{Cueva Grande}

En este yacimiento se realizaron dos pozos de sondeo debido al potencial estratigráfico que ofrece, a juzgar por la evidencia de los pozos de "huaqueo" previos.

De esta manera, el pozo 1 (fig. 7b) se ubicó en la cámara principal, a 4,5 $\mathrm{m}$ de la línea de goteo y a 1,6 metros de la pared O de la cueva, en medio de dos claras excavaciones ilegales. Los trabajos desarrollados 
permitieron documentar un denso conchal con una potencia no determinada, pero que supera los $1,5 \mathrm{~m}$ de espesor. El reducido tamaño de la unidad de sondeo impidió alcanzar la roca base de la cueva. El conchal se encuentra cubierto por dos estratos que sellaron la ocupación y que no permitieron, al menos en este sector, una alteración significativa por parte de los saqueadores.

El estrato o capa 1 presenta una matriz carbonosa con abundante conchilla molida, la que forma parte de los sedimentos removidos por los "huaqueros" que alcanzaron tangencialmente el área de excavación. Esta capa posee entre 2 y $5 \mathrm{~cm}$ de potencia, y es más espesa hacia el norte de la cueva.

Bajo esta se registra el estrato 2, que corresponde a una depositación arqueológicamente estéril y compuesta por una matriz limosa de coloración marrón anaranjado. Esta se intercala con al menos seis lentes de gravilla color grisáceo de escasos centímetros de potencia, los que se disponen de forma discontinua. La ausencia de clastos angulares provenientes del derrubio permite suponer que el origen de este estrato sería eólico. La capa 2 se extiende entre los 2 y $40 \mathrm{~cm}$ de profundidad, y su potencia aumenta hacia el sur de la unidad, situación que sugiere una depositación de los estratos culturales suprayacentes con pendiente hacia el exterior de la cueva, pero ello no es visible en el piso actual. En la interfaz entre los estratos 2 y 3 , en la porción No del pozo, se registró un rasgo carbonoso de no más de $5 \mathrm{~cm}$ de potencia que correspondería a un último evento de combustión antes del abandono definitivo del yacimiento. Descartamos la alternativa de que evidencie ocupaciones modernas, ya que -como hemos dicho- en los estratos suprayacentes no se advierten conchas ni restos arqueológicos

El conchal comienza inmediatamente bajo el estrato estéril, en torno a los $30 \mathrm{~cm}$ de profundidad, y se extiende por lo menos hasta los $180 \mathrm{~cm}$; los motivos logísticos explicados antes impiden una delimitación exacta de su potencia. A partir de la completitud de las valvas y de la variación en la preponderancia de las especies presentes, en terreno el conchal fue dividido en tres grandes capas.

La capa 3a presenta una matriz arenosa de color negruzco y con valvas, en su mayoría, fragmentadas, aunque con un aporte importante de ejemplares completos o casi completos. Se registra una gran cantidad de guijarros redondeados de tamaños que oscilan entre $5 \mathrm{y}$ a

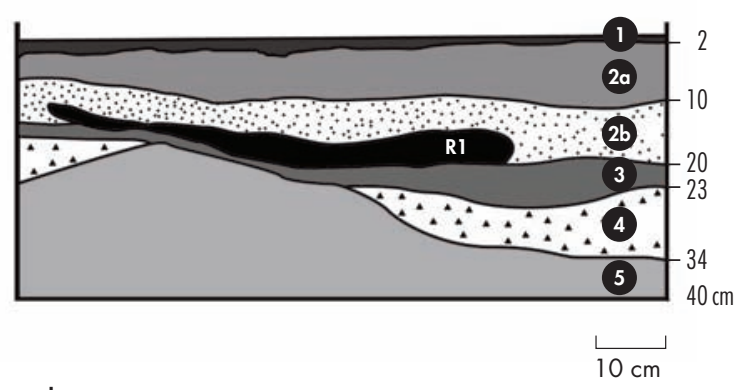

b

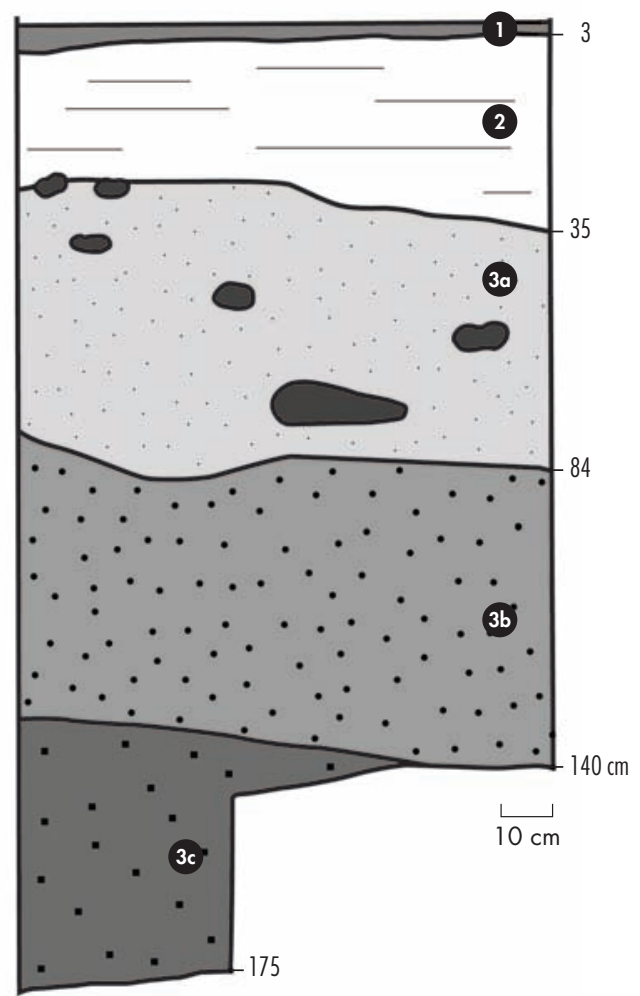

Figura 7. Estratigrafía de las cuevas del morro Vilcún: a) pozo 1 de la cueva Mediana, perfil este; b) pozo 1 de la cueva grande, perfil este. Figure 7. Stratigraphy of Morro Vilcún caves: a) pit 1 of the Medium cave, eastern profile; $\boldsymbol{b}$ ) pit 1 of the Large cave, eastern profile.

$20 \mathrm{~cm}$, dispuestos aleatoriamente o formando pequeñas concentraciones. Algunos de ellos exhiben claras huellas de hollín, mientras que otros se encuentran fracturados por termofractura, ya que no se aprecian puntos de impacto. Los moluscos recuperados provienen sobre todo de ambientes rocosos; los principales son: loco, tégula, maucho (Nacella sp.), lapas (al menos dos especies) $\mathrm{y}$, en menor medida, mitylidos, caracoles (Acantina 
sp., Nassarius gayi, Argobuccium sp.), poliplacóforos, cirripedios, decápodos y echinoideos. No obstante, se identificaron valvas de almeja (Protothaca thaca), lo que indica acceso a recursos de fondo arenoso. Por otra parte, se recuperaron restos de peces, aves y, en menor medida, carnívoros y roedores. Este estrato se extiende entre los 31 y $84 \mathrm{~cm}$ de profundidad. En el nivel $9(80-90 \mathrm{~cm})$ se documentaron tres importantes variaciones que definen la capa $3 \mathrm{~b}$ : la marcada disminución de guijarros, la mayor fragmentación de las valvas y la baja en la frecuencia de locos en el conchal, dominado en cambio por lapas, mauchos y tégulas. La diversidad de moluscos no varía de manera significativa, aunque la densidad de conchilla hace suponer un uso más intensivo del espacio. Mientras los restos de peces aumentan, disminuyen los de aves y mamíferos, encontrados en la capa superior. Entre los 130 y 140 $\mathrm{cm}$ de profundidad se observa una disminución en la compactación del sustrato, asociada con la aparición de lapas, tégulas y mauchos prácticamente completos, lo que marca el inicio de la capa 3c. Este estrato se mantiene hasta el cierre de la unidad, a los $180 \mathrm{~cm}$ de profundidad. Una muestra de carbón obtenida de la esquina No a los $178 \mathrm{~cm}$ arrojó un fechado absoluto de $750 \pm 30$ AP (UGAMS 11722).

El pozo 2 se emplazó bajo el conjunto de grabados 6 y 7 , en el inicio del pasillo posterior de la cueva, al interior de una depresión interpretada como una excavación ilegal, con la esperanza de que los depósitos más profundos se encontraran inalterados. Por desgracia, ello no fue así, y el hallazgo de elementos modernos hasta una profundidad de $60 \mathrm{~cm}$ motivó la interrupción de la excavación. En efecto, se identificó un estrato superior (capa 1) compuesto por una matriz poco compacta, dominada por valvas de lapa, mitylidos, caracoles, locos y restos de erizos muy poco fragmentados. De él se recuperaron restos óseos de pinnípedos, peces y aves, pero también material histórico/subactual, como restos de género y un pequeño botón quemado proveniente del nivel 5. En los niveles 3 y 4 es importante mencionar el hallazgo de restos óseos humanos, correspondientes a dos sesamoideos y un incisivo sin su raíz, lo que daría cuenta de un contexto funerario y de la presencia de un individuo juvenil.

Hacia los $40 \mathrm{~cm}$ de profundidad, y concentrado en la porción sur de la unidad, se registró un segundo estrato, el que se torna homogéneo en toda la unidad a los $55 \mathrm{~cm}$ de profundidad; está compuesto por una matriz
Tabla 2. Resumen de restos óseos identificados (NISP) de los tres sondeos estratigráficos practicados en el morro Vilcún: P1 CG) pozo 1 de la cueva Grande; P2 CG) pozo 2 de la cueva Grande; P1 CM) pozo 1 de la cueva Mediana. Table 2. Summary of bone remains identified (NISP) in three stratigraphic test pits performed at Morro Vilcún, P1 CG) Pit 1 in Large Cave; P2 CG) Pit 2 in Large Cave; P1 CM) Pit 1 in Medium Cave.

\begin{tabular}{l|r|r|r|r} 
Taxa & P1 CG & P2 CG & P1 CM & Total \\
Otariidae & 3 & & & 3 \\
\hline Carnivora & 1 & & & 1 \\
\hline Rodentia & 1 & 3 & 3 & 7 \\
\hline H. sapiens & 2 & 1 & & 3 \\
\hline Mammalia & 2 & & & 2 \\
\hline Aves & 19 & & & 19 \\
\hline Osteichthyes & 430 & 8 & 3 & 441 \\
\hline Total & 458 & 12 & 6 & 476
\end{tabular}

Tabla 3. MNI de peces del pozo 1 de la cueva Grande. Table 3. MNI of fish from Test Pit 1 of Large Cave

\begin{tabular}{l|c|c|c|c|c|c} 
Taxa & \multicolumn{5}{|c}{ Estratos } \\
& 1 & 2 & $3 \mathrm{a}$ & $3 \mathrm{~b}$ & $3 \mathrm{c}$ & Total \\
\hline Pez. Ind & & 1 & 2 & 3 & 2 & 8 \\
\hline Bilagay & & & 1 & & 2 & 3 \\
\hline Cabrilla & & & 2 & 3 & 2 & 7 \\
\hline Congrio & & & & 2 & 1 & 3 \\
\hline Jurel & & & 1 & 1 & 1 & 2 \\
\hline Merluza & & & 1 & 11 & 4 & 20 \\
\hline Róbalo & 1 & & 4 & 11 & 12 & 45 \\
\hline Tomollo & & & 1 & 10 & 12 \\
\hline Total & 1 & 1 & & & \\
\hline
\end{tabular}

de limo y arena color marrón, comparativamente más compacta. A juzgar por la composición estratigráfica, es posible que se relacione con la depositación estéril ubicada en la parte anterior de la cueva, denominada estrato 2 en el pozo 1. 


\section{Evidencias culturales}

Los restos malacológicos no fueron objeto de ninguna recolección sistemática, por lo que el análisis zooarqueológico se restringe a elementos de vertebrados. Los restos óseos ascienden a 581 especímenes, de los cuales se identificaron (NISP) 476 (81,9\%). En lo fundamental, provienen del pozo 1 de la cueva Grande (96,2\%); en las restantes unidades recuperó muy poco material identificable (tabla 2). Por lo anterior, los resultados faunísticos que se ofrecen aquí provienen solo de la primera de las unidades. La muestra del pozo 1 de la cueva Grande se encuentra ampliamente dominada por restos de peces (tabla 2), seguidos muy atrás por aves aún no identificadas y mamíferos (otáridos, cánidos y roedores).

Respecto de los peces, se identificaron un total de ocho taxa: róbalo (Eleginops maclovinus), tomollo (Auchenionchus variolosus), cabrilla (Sebastes capensis), jurel (Trachurus murphyi), bilagay (Cheilodactylus variegatus), congrio negro (Genypterus maculatus), merluza (Merluccius gayi) y sierra (Thysrsites atun). No obstante, cerca de un $40 \%$ corresponde al róbalo. A este le siguen la cabrilla (14\%) y el congrio (8\%); las restantes especies poseen representaciones menores. Las especies reconocidas habitan tanto en fondos arenosos como rocosos, ambos presentes en las inmediaciones del morro (Mann 1954). En total, se estimó un número mínimo de 45 ejemplares, sobre todo róbalos (tabla 3 ). En términos estratigráficos, el depósito más fértil del sitio es la capa $3 \mathrm{~b}$, tanto en términos de restos identificados como en individuos, aunque se debe considerar que la capa $3 \mathrm{c}$ se encuentra menos representada en la excavación. En la capa $3 \mathrm{a}$ y b predominan individuos de fondos arenosos sobre aquellos que habitan fondos rocosos (ca. 70\% contra 30\%), mientras que en la capa $3 c$ esta proporción se invierte (ca. 60 contra 40\%). La frecuencia de elementos esqueletales es variada y señala que los ejemplares eran traslados completos a la cueva Grande para su procesamiento, consumo y descarte (tabla 4).

El resto del conjunto faunístico se completa con 19 restos de aves (al menos dos especies), una falange y fragmentos de un astrágalo de Otariidae indet., una vértebra lumbar y un canino de zorro (Lycalopex sp.), y una costilla y un fragmento de maxilar de un roedor indeterminado.

Las modificaciones naturales son escasas en la muestra, y se observan marcas de abrasión, pisoteo y meteorización, aunque en proporciones que no superan
Tabla 4. Frecuencia de partes esqueletarias de peces identificados en el pozo 1 de la cueva Grande. Table 4. Frequency of skeletal parts of fish identified in Test Pit 1 of the Large Cave.

\begin{tabular}{l|r|r|r} 
Elementos & NISP & MNE & MAU \\
Articular & 25 & 25 & 12,5 \\
\hline Atlas & 2 & 2 & 2 \\
\hline Branquial & 1 & 1 & 1 \\
\hline C. Supraoccipital & 1 & 1 & 1 \\
\hline Cleitro & 38 & 20 & 10 \\
\hline Costilla & 33 & 33 & - \\
\hline Cuadrado & 16 & 15 & 7,5 \\
\hline Dentario & 27 & 25 & 12,5 \\
\hline Hiomandibular & 17 & 16 & 8 \\
\hline Infrabranquial & 2 & 2 & 2 \\
\hline Maxila & 33 & 33 & 16,5 \\
\hline Opérculo & 9 & 8 & 4 \\
\hline Palatino & 18 & 18 & 9 \\
\hline Posttemporal & 1 & 1 & 0,5 \\
\hline Premaxila & 7 & 6 & 3 \\
\hline Preoperculo & 27 & 15 & 7,5 \\
\hline Supracleitro & 10 & 5 & 2,5 \\
\hline Urohial & 10 & 10 & 5 \\
\hline Vert. Caudal & 71 & 71 & \\
\hline Vert. Precaudal & 19 & 18 & \\
\hline Vert. N/D & 71 & 45 & \\
\hline Vómer & 4 & 4 & 4 \\
\hline
\end{tabular}

el 15,6\% en el caso de esta última. Por su parte, las marcas culturales remiten exclusivamente a la termoalteración, la cual sin embargo afecta a solo un 2,3\% del total, presente tanto en huesos de peces como en aves.

El universo lítico trabajado es muy reducido. En la capa 3 del pozo 1 de la cueva Grande, se registraron solo tres espesas lascas primarias en rocas ígneas de posible procedencia local, una de ellas con evidencias de microastillado en uno de sus bordes, seguramente por uso. A ello se suma a un guijarro subcilíndrico (ca. $10 \mathrm{~cm}$ de largo) con negativos de percusión en ambos extremos (fig. 8a). Finalmente, destaca la presencia de 
una pequeña cuenta lítica de $0,4 \mathrm{~cm}$ de largo con una perforación bicónica (fig. 8b). Por su parte, en la cueva Mediana se documentó una sola lasca primaria de tamaño medio (ca. $5 \mathrm{~cm}$ de largo) en la capa $2 \mathrm{~b}$.

Los materiales cerámicos recuperados corresponden a dos fragmentos provenientes de la cueva Mediana, capa $2 \mathrm{~b}$ (fig. 8c). El primero de estos es una porción de cuerpo de 48,8 x 40,9 mm y 7,8 mm de espesor. En ambas caras presentan tratamiento de superficie pulido y engobe del mismo color de la pasta (blanquecino grisáceo), así como exposición al fuego. Sobre el engobe blanquecino de la cara interior, se despliega un campo de pintura roja formando un diseño indeterminado. Por su parte, el fragmento $n^{\circ} 2$ es una porción de cuerpo 24,7 x 19,5 $\mathrm{mm}$ y $7,1 \mathrm{~mm}$ de espesor. Al igual que el fragmento ${ }^{\circ}$ 1 , posee tratamiento de superficie pulido por ambas caras y engobe del mismo color de la pasta (blanquecino grisáceo). Por el interior y sobre el engobe se observan restos muy débiles de pintura roja; en esa misma cara se advierte también exposición al fuego.

Es muy probable que ambos fragmentos pertenezcan a la misma pieza, la cual pudo haber tenido morfología abierta. Ambos presentan un tipo de pasta compuesta por una matriz arcillosa compacta de color blanquecino, con abundantes restos de biotitas y cuarzos, lo que da cuenta de formaciones graníticas como parte del antiplástico. A su vez, se registran posibles inclusiones volcánicas. Esta pasta podría analogarse con lo que ha sido definido como familia granítica-volcánica, registrada en distintos puntos del área norpatagónica, como por ejemplo en el Alfarero Tardío Calafquén (Reyes et al. 2003-2004) y Alfarero Tardío de la costa de Valdivia (Adán et al. 2007). De igual manera, los materiales son comparables a los encontrados en Yaldad (Legoupil 2005) y otros contextos en Chiloé (Alvarez et al. 2008). La forma, tratamiento de superficie y decoración no corresponden a nada descrito como posthispano, que por lo general es monocromo, aunque se ha documentado el caso de tradiciones alfareras prehispánicas en uso, sobre todo, por grupos rurales (Hajduk 1981-82; Bustos 2005).

\section{DISCUSIÓN}

Aunque limitadas en sus pretensiones, nuestras investigaciones no solo aportan datos significativos en el marco del mínimo conocimiento previo de la prehistoria regional, sino que además abren una serie de interrogantes a

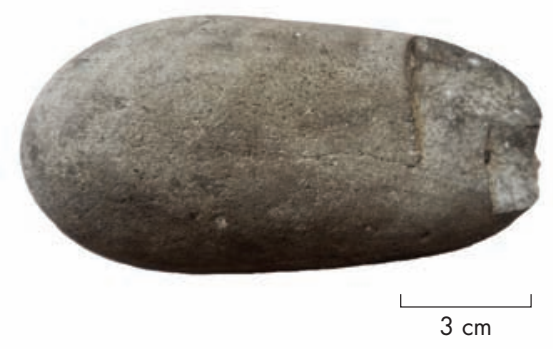

b

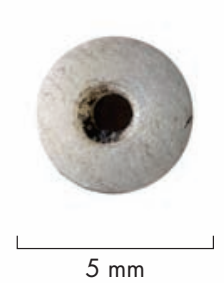

c

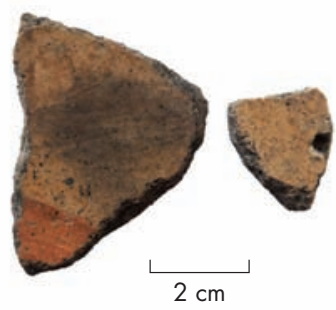

Figura 8. Materiales culturales de las cuevas del morro Vilcún: a) guijarro con percusión bipolar; b) cuenta lítica con perforación bicónica; c) fragmentos cerámicos. Figure 8. Cultural materials found in the Morro Vilcún caves: a) pebble with percussion marks on each end; b) perforated biconic stone bead; c) ceramic sherds.

arqueológicas para ser resueltas en futuros trabajos.

En lo que respecta a la cronología, tenemos certeza de que las cuevas fueron habitadas hace más de ochocientos años. Sin embargo, es necesario señalar que no pudimos alcanzar la base de los depósitos en la cueva Grande y tan solo se fecharon carbones (con el fin de evitar las confusiones asociadas al efecto reservorio, desconocido en la zona). Por el momento, las fechas absolutas no pueden ser vinculadas directamente con las pinturas rupestres, y se limitan a dar cuenta de la antigüedad de los depósitos estratigráficos.

Si bien es cierto que de la base de la cueva Mediana se obtuvo una fecha histórica, lo más probable es que ello refleje perturbaciones estratigráficas producto de actividades domésticas relativamente recientes, las que habrían alterado componentes más tempranos poco densos, como los representados por los fragmentos de cerámica de posible filiación Alfarera tardía, los que por lo demás se recuperaron por encima de la muestra fechada. Asimismo, es dable suponer incluso que haya habido una intencionalidad clara de excavar o buscar "tesoros" (y tal vez entierros humanos) por parte de grupos de pescadores o hacheros chilotes que frecuentaban estas costas antes de que hubiera asentamientos o poblados "occidentales" (Urbina 2011). La datación absoluta de 


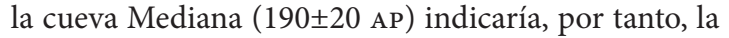
antigüedad de parte de las perturbaciones ocurridas en ella y que han continuado hasta la actualidad, como lo refleja el graffiti y la basura moderna en superficie y capas superiores.

Los sitios con fechados absolutos en el área son escasos, y en cambio los esfuerzos se concentran en la datación de las capas más tempranas, con el objeto de documentar las primeras adaptaciones marítimas del área (Porter 1992; Gaete \& Navarro 2004; Ocampo \& Rivas 2004; Legoupil 2005). Varios de estos sitios presentan fragmentos cerámicos en sus niveles superiores. Algunos de estos, datados por termoluminiscencia, sugirien que grupos portadores de cerámica se habrían instalado en el área al menos desde el 700 DC (Munita et al. 2012). En los conchales de Piedra Azul y Huenquillahue 2 se han obtenido dataciones prehispánicas contemporáneas a las ocupaciones registradas en la cueva Grande (Gaete \& Navarro 2004; Munita et al. 2012). Un fechado absoluto de los materiales cerámicos de Vilcún podrá arrojar luz sobre esta discusión cronológica.

Por último, la ausencia de cerámica en la cueva Grande, y su presencia en la cueva Mediana, podría deberse a las distintas actividades desarrolladas en cada una, si se asume que ambas son contemporáneas arqueológicamente.

En lo que respecta a subsistencia, es claro que, al menos en la cueva Grande (y probablemente en la cueva Alta), se consumió una variedad de moluscos y crustáceos, y en menor cantidad peces y aves. La presencia de mamíferos terrestres es marginal y los restos de roedores se explicarían por introducciones naturales al sitio (perchas o fecas). Por otro lado, se observa una evidente adaptación al medio costero (lo que no necesariamente implica navegación) a partir de la amplia cantidad de individuos ictiológicos presentes en el sitio, además del ya referido conchal. Dentro de las taxa ictiológicas, predominan las especies cuyo hábitat se emplaza más cerca de la costa o bien que migran hacia la misma en algún momento. Todos los peces identificados se encuentran próximos al área, pero se diferencian entre aquellos que prefieren suelos de tipo rocoso y los que buscan superficies más arenosas. Dentro de las especies correspondientes a suelos arenosos se encuentran el róbalo, el jurel, la sierra y la merluza, todos los cuales se mueven en cardumen. Por su parte, aquellas especies asociadas a sustratos rocosos son el bilagay, la cabrilla, el congrio negro y el tomollo; todas además se caracterizan por hábitos más solitarios. Al respecto, resulta interesante la presencia del tomollo en la capa $3 c$ del pozo 1 de la cueva Grande, ya que en la actualidad su límite de distribución por el sur va desde el sector del seno de Reloncaví y áreas aledañas hasta la Isla Grande de Chiloé, debido a factores relacionados con las propiedades de las corrientes de agua y sus consiguientes cadenas tróficas (Sáez \& Pequeño 2009). Su presencia en el contexto de la cueva Grande podría interpretarse como un eventual indicador de movilidad humana, aunque no descartamos la posibilidad de que variaciones en las corrientes marinas en el pasado hayan generado una migración del tomollo al área del morro Vilcún.

Para la obtención de aquellas especies que se mueven en cardumen, el arte de pesca más apropiado es el empleo de redes o también la implementación de corrales de pesca; dado el ambiente del área de estudio, esta última parece la más apropiada (Álvarez et al. 2008). Asimismo, la amplia cantidad de róbalos, en comparación con los jureles, sugiere que esta última fue capturada a modo de fauna acompañante, mientras que la presencia de la merluza señalaría una movilidad hacia aguas más adentro e implicaría el uso de embarcaciones. En cuanto a las especies de sustratos rocosos, su captura se vincula con líneas de pesca, canastas o bien arpones, los que sin embargo no fueron documentados en los sitios estudiados.

Los instrumentos y desechos líticos son bastante escasos, a tal punto de que en la cueva Grande se recuperaron solo cuatro piezas, mientras que en la cueva Mediana se identificó un desecho primario. Se trata de una tecnología expeditiva, conformada por desechos primarios y guijarros con percusiones que estarían implicados en el procesamiento de moluscos y peces. Aunque sin huellas de modificaciones antrópicas explícitas, la recurrente presencia de guijarros redondeados en la cueva Mediana revela su traslado cultural al sitio, en razón de hipotéticas prácticas culinarias ("curantos"), como lo sugiere la presencia de hollín y lo que parecen ser termofracturas en algunos de ellos. Por último, la excepcional presencia de una pequeña cuenta lítica en la cueva Mediana señala sin duda la presencia de elementos ornamentales.

Es más difícil aventurar interpretaciones con respecto a la "función" o actividades desarrolladas en los sitios, aunque la evidencia recuperada es consistente con la interpretación de un uso "ritual" o "ceremonial" 
comunitario reiterado, que implicó la preparación y consumo de grandes cantidades de mariscos y, en menor cantidad, peces, como lo sugieren las piedras de curanto. No se recuperaron artefactos ni evidencias de actividades domésticas continuas, aunque ello puede reflejar un sesgo por haber excavado menos de un $1 \%$ de la superficie en ambas cuevas. Primero Bird (1988) y más tarde Legoupil (2005) documentaron en la Isla Grande de Chiloé conchales con una estructura muy similar al de la cueva Grande: muy espesos pero con predominancia casi exclusiva de restos de moluscos y crustáceos, baja frecuencia de vertebrados, ausencia de lobos marinos y evidencia de escasos instrumentos líticos. La presencia de sitios comparativamente más variados, como Puente Quilo 1 (Ocampo \& Rivas 2004) y conchal Gamboa (Díaz \& Garretón 1972-73), hace suponer que lo registrado en la cueva Grande de Vilcún se relacionaría con áreas de descarte específicas, por lo que se podría encontrar campamentos en las cercanías (Legoupil 2005). Por su parte, la información que disponemos para la cueva Mediana es escasa, y solo la presencia de posible cerámica prehispánica inserta en delgados estratos con conchilla molida apuntaría a la realización de mínimas actividades domésticas.

La pregunta acerca de su filiación étnica solo nos permite señalar que se trata de grupos costeros, muy probablemente canoeros (p. e. emplazamiento frente al mar, escasez de recursos terrestres, difícil acceso y transitabilidad por tierra, facilidades de transporte de grandes volúmenes y pesos, presencia de peces de aguas profundas cuya captura requiere navegación, semejanzas con contextos insulares). Sin mayores fechados ni elementos estilísticamente diagnósticos, no podemos siquiera especular si se trata de canoeros, y menos aun si estamos frente a chonos o alguna de las muchas otras parcialidades descritas en tiempos coloniales (Álvarez 2002).

En términos muy generales, las pinturas de Vilcún son comparables a las registradas en contextos canoeros, como las de la cueva Pacífico de isla Madre de Dios (líneas y puntos en rojo, sin superponer, motivo de círculo con trazos divergentes) (Centre Terre 2006; Maire et al. 2009); aún más al sur, podrían presentar afinidades con las del del alero Martín González, en el oeste de la isla Hoste, en Tierra del Fuego (manchones y líneas en color rojo) (González et al. 2014). Es probable que estas coincidencias se deban a la simplicidad formal de los motivos, puesto que ambos sitios están separados de Vilcún por más de mil kilómetros.
Este tipo de motivos simples se encuentran también en contextos terrestres, como los descritos por Castelleti (2007) en Renahue 2 (lago Caburgua); sin embargo, su estructura y configuración es distinta, puesto que poseen una mayor estandarización y se asocian sobre todo a tridígitos. Por otro lado, creemos que las pinturas del morro Vilcún no son comparables con la modalidad del Estilo de Grecas (Menghin 1957) de los bosques montanos en el lago Nahuel Huapi y otros cuerpos lacustres vecinos, definida como MALB por Albornoz y Cúneo (2000), debido a la mayor diversidad y complejidad en los motivos y las sensibles diferencias en las configuraciones espaciales. Con todo, el hallazgo de un posible positivo de mano muy deslavado (fig. 5b) podría también interpretarse como una mera coincidencia, pero es sugerente de algún tipo de contacto y/o conocimiento remoto con cazadores terrestres del interior.

Respecto de los grabados de la cueva Grande, los motivos circulares o subcirculares con trazos en su interior recuerdan a las "vulvas" registradas en contextos rupestres norteños, como aquellos del área costera de Valdivia (Adán \& Godoy 2006), Malleco (Berdichewsky 1968) y Llaima (Oyarzún 1979), lo que sugiere relaciones con el área mapuche.

Por último -y aunque no podamos siquiera afirmar que las pinturas y grabados en las diferentes cuevas sean contemporáneos-, saltan a la vista ciertos patrones y oposiciones: escaso depósito cultural en la cueva Mediana contra un denso conchal en la cueva Grande; ausencia de pinturas en el interior oscuro de la cueva Alta; casi total ausencia de pinturas en la parte oscura de la cueva con grabados y total ausencia de grabados en aquella con más pinturas, que resulta ser además la más iluminada. Estos podrían dar luces del sistema de creencias y/o "ritualidad" de estos pueblos y esperamos sean susceptibles de interpretación gracias a futuros hallazgos.

\section{CONCLUSIONES}

Los trabajos desarrollados en el morro Vilcún entregaron importante información referente a aspectos poco conocidos de la prehistoria de los canales norpatagónicos. Se documentaron cuatro cuevas, tres de las cuales presentan arte rupestre: principalmente, pinturas de líneas, puntos y manchones en color rojo, las que se concentran en la cueva Mediana. Aun cuando se trata 
de motivos muy simples y presentes en contextos de grupos cazadores terrestres contemporáneos, creemos que la disposición de los motivos (formando unidades discretas) y la ausencia de representaciones complejas (conjuntos de motivos) conforman una suerte de "estilo" particular. Sin embargo, se debe conducir nuevos trabajos de campo para contar con una muestra mayor que permita contrastar esta hipótesis. No obstante lo anterior, la posible presencia de un positivo de mano y de "vulvas" sugiere algún contacto con grupos cazadoresrecolectores del interior y también del territorio mapuche, ubicado más al norte. En términos cronológicos, hay evidencia de que al menos la cueva Grande fue utilizada hace 800 años de manera muy intensa, mientras que la cueva Mediana exhibiría una ocupación prehispánica tardía efímera, alterada por actividades históricas. Estas perturbaciones "modernas" (si bien algunas son anteriores al establecimiento de colonos y poblados "chilenos" en este territorio) reflejan una realidad que es demasiado común: que los yacimientos arqueológicos suelen ser conocidos mucho antes de que los científicos sepan siquiera de su existencia. Una adecuada colaboración entre la comunidad local y los investigadores, por lo tanto, puede evitar alteraciones innecesarias ( $\mathrm{y}$ en algunos casos incluso su destrucción) y contribuir, en cambio, a su registro sistemático, publicación e investigación. La labor de investigación científica y la patrimonial debieran ser complementarias, como revela el que un proyecto FONDART como este no termine tan solo con una bella exposición fotográfica y charlas de educación patrimonial a escolares, sino que se difunda en publicaciones académicas. De este modo, esperamos que este proyecto (seguramente beneficiado por tratarse de un hallazgo llamativo, como son las pinturas y grabados rupestres en cuevas localizadas en un paisaje sobrecogedor) sea apenas un primer paso, tanto para la puesta en valor de estos sitios, como para futuras investigaciones arqueológicas (p. e. prospecciones, excavaciones ampliadas, estudios paleoambientales) en esta zona poco conocida del país.

RECONOCIMIENTOS Esta investigación fue financiada por el proyecto FONDART regional Folio 7924. Agradecemos a la Municipalidad de Chaitén por la ayuda prestada, así como a la escuela Almirante Juan José Latorre, en especial a su director Raúl Jara y al profesor Luis Soto. Verónica Reyes revisó los materiales cerámicos. Álvaro Calás realizó la digitalización de las figuras. Finalmente, un especial reconocimiento a la comunidad de Chaitén por la gran acogida a nuestro proyecto de difusión.

\section{REFERENCIAS}

AdÁn, L. \& M. Godoy, 2006. Huellas de historia: patrimonio cultural de la reserva costera valdiviana. Valdivia: Imprenta Austral.

Adán, L.; R. Mera, F. Bahamondes \& S. Donoso, 2007. Historia cultural de la cuenca de Valdivia: proposiciones a partir del estudio de sitios alfareros prehispánicos e históricos. Revista Austral de Ciencias Sociales 12: 5-30.

Albornoz, A. M. \& E. Cúneo, 2000. Análisis comparativo de sitios con pictografías en ambientes lacustres boscosos de Patagonia septentrional. En Arte en las rocas. Arte rupestre, menhires y piedras de colores en Argentina, M. M. Podestá \& M. de Hoyos, Eds., pp. 163-174. Buenos Aires: Sociedad Argentina de AntropologíaAsociación de Amigos del INA.

Álvarez, R., 2002. Reflexiones en torno a las identidades de las poblaciones canoeras situadas entre los $44^{\circ}$ y los $48^{\circ}$ de latitud sur, denominadas chonos. Anales del Instituto de la Patagonia Serie Ciencias Humanas 30: 79-86.

Álvarez, R.; D. Munita, J. Fredes \& R. Mera, 2008. Corrales de pesca en Chiloé. Valdivia: Imprenta Americana.

ANDREFSKY, W., 2006. Lithics: Macroscopic approaches to analysis. Cambridge: Cambridge University Press.

Berdichewsky, B., 1968. Excavaciones en la cueva de Los Catalanes. Boletín de Prehistoria de Chile 1: 33-83. Santiago: Universidad de Chile.

BIRD, J., 1988. Travels and archaeology in south Chile. Iowa: University of Iowa Press.

Bustos, C., 2005. Reconstrucción de las tradiciones alfareras de Caulín y Apiao a partir de fuentes orales. Chiloé, $\mathrm{x}^{\mathrm{a}}$ región. Tesis para optar al grado de Licenciado en Antropología, Universidad Austral, Valdivia.

CAstelleti, J., 2007. El arte rupestre de la zona boscosa y lacustre cordillerana del sur de Chile y sus relaciones con regiones vecinas. Boletín de la Sociedad Chilena de Arqueología 40: 57-71.

Centre Terre 2006. Última Patagonia <http://www.centre-terre. fr/ultima2006/UltimaPatagonia_CRfevrier.PDF> [Consultado 11-10-2016].

DíaZ, C. \& M. GARretón, 1972-1973. El poblamiento prehispánico del área insular septentrional chilena. En: Actas del vi Congreso de Arqueología chilena, pp. 559-584. Santiago: Universidad de Chile.

EQUiPo PAT, 2012. Rumores populares, hallazgos científicos. Revista PAT 53: 32-40. <http://www.revistapat.cl/numeros/PAT53/index. html\#32/z> [Consultado 11-10-2016].

Falabella, F.; R. Meléndez \& L. Vargas, 1995. Claves osteológicas para peces de Chile central: un enfoque arqueológico. Santiago: Artegrama.

Gaete, N. \& X. Navarro, 2004. Estrategias de vida de canoeros cazadores pescadores recolectores del seno de Reloncaví. Entre el bosque siempreverde y el mar interior. Región de Los Lagos, Chile. En Contra viento y marea. Arqueología de Patagonia, M. Civalero, P. Fernández \& A. Guráieb, Eds., pp. 217-235. Buenos Aires: INAPLA-Sociedad Argentina de Antropología.

GajARdo, R., 1994. La vegetación natural de Chile: clasificación y distribución geográfica. Santiago: Universitaria.

González, M.; M. Gañán \& A. Serrano, 2014. Primer registro de arte rupestre en Tierra del Fuego. Magallania 42 (2): 175-181.

Gradín, C., 1978. Algunos aspectos del análisis de las manifestaciones rupestres. Revista del Museo Provincial 1: 120-137.

Grayson, D., 1984. Quantitative Zooarchaeology. Orlando: Academic Press. 
HajDuk, A., 1981-1982. Cementerio "Rebolledo Arriba" departamento Aluminé, provincia de Neuquén. Relaciones de la Sociedad Argentina de Antropología 14 (2): 125-145.

Harman, J., 2016. DStretch. A tool for the digital enhancement of pictographs. <www.dstretch.com $>$ [Consultado 11-10-2016].

LegoupIL. D. 2005. Recolectores de moluscos tempranos en el sureste de la isla de Chiloé: una primera mirada. Magallania 33 (1): 51-61.

Leroi-Gourhan, A., 1968. Prehistoria del arte occidental. Barcelona: Gustavo Gili.

Lyman, R., 1994. Vertebrate taphonomy. Cambridge: Cambridge University Press.

Maire, R.; B. Tourte, S. Jaillet, J. Despain, B. Lans, F. Brehier, L. Fage, L. Morel, M. Pouilly, T. Datry, M. Massaul, D. Genty, K. Wainer, V. Ridoux, W. Dabin, J. Pernette, M. Agüero \& M. J. MANNESCHI, 2009. Geomorphic and archaeological features of coastal caves on Madre de Dios archipelago (Patagonia, Chile). Proceedings of 15th International Congress of Speleology, Kerrville, Texas. <http://www.centre-terre.fr/ultima2010/ressources/Documents\%20en\%20anglais/Coastal\%20caves\%20of\%20Madre\%20 de\%20Dios.pdf> [Consultado 11-10-2016].

Mena, F.; E. González \& R. Labarca, 2011. Primeros registros de arte rupestre en el litoral septentrional de la Patagonia chilena. Magallania 39 (2): 303-307.

Menghin, O., 1957. Estilos de arte rupestre de Patagonia. Acta Praehistórica I: 57-87.

Munita, D.; R. Mera, I. Arregui \& M. Manneschi, 2012. Funebria de grupos canoeros durante el Holoceno tardío en la región de los Lagos. El conchal de Yaco Alto -1, Calbuco, Chile. CazadoresRecolectores del Cono Sur. Revista de Arqueología 6: 17-41. La Plata, Argentina.
Ocampo, C. \& P. Rivas, 2004. Poblamiento temprano de los extremos geográficos de los canales patagónicos: Chiloé e Isla Navarino 1. Chungara 36 (supl.): 317-331.

Oyarzún, A., 1979. Los petroglifos del Llaima. En Estudios Antropológicos y Arqueológicos, M. Orellana, Comp., pp. 58-64. Santiago: Universitaria.

Podestá, M. M.; R. Paunero \& D. Rolandi, 2005. El arte rupestre de Argentina indígena: Patagonia. Buenos Aires: Casano Gráfica Ltda.

Porter, Ch., 1992. Gua-010, un sitio costero erosionado en una zona sísmica activa. En Actas del xII Congreso Nacional de Arqueología chilena. Tomo I, Simposios. Boletín del Museo Regional de la Araucanía, 81-94.

Reyes, V.; L. SAnhueza \& L. AdÁn, 2003-2004. Alfarería doméstica y funeraria de la región de Calafquén. Revista de Antropología 17: 151179. < http://repositorio.uchile.cl/bitstream/handle/2250/122013/ Sanhueza_RN_007_2003-2004.pdf?sequence=18isAllowed=y > [Consultado 11-10-2016].

Rice, P., 2006. Pottery analysis: A sourcebook. Chicago: University of Chicago Press.

SÁez, S. \& G. Pequeño, 2009. Clave taxonómica, actualizada, ilustrada y comentada de los peces de la familia Labrisomidae de Chile (Perciformes, Blennioidei). Gayana 73 (1): 130-140.

Urbina, X., 2011. Análisis histórico-cultural del alerce en la Patagonia septentrional occidental, Chiloé, siglos xvi al xix. Magallania 39 (2): $57-73$. 